\title{
On a class of sequential fractional
} $q$-integrodifference boundary value problems involving different numbers of $q$ in derivatives and integrals

\author{
Nichaphat Patanarapeelert, Umaporn Sriphanomwan and Thanin Sitthiwirattham*
}

\section{"Correspondence:}

thanin.s@sci.kmutnb.ac.th Nonlinear Dynamic Analysis Research Center, Department of Mathematics, Faculty of Applied Science, King Mongkut's University of Technology North Bangkok, Bangkok, Thailand

\begin{abstract}
In this paper, we study a sequential fractional $q$-integrodifference equation with fractional $q$-integral and $q$-derivative boundary value conditions. Our problem contains two different fractional orders and six different numbers of $q$ in derivatives and integrals. By using Banach's contraction principle and Krasnoselskii's fixed point theorem, some new existence and uniqueness results are obtained. An illustrative example is also presented.
\end{abstract}

MSC: 39A05; 39A13

Keywords: existence; $q$-derivative; $q$-integral; $q$-integrodifference equation

\section{Introduction}

In the 20th century, the intensive works on $q$-difference equations have became interesting subject of research work [1-3] in the areas of mathematics and applications such as the applications to orthogonal polynomials and mathematical control theories. Basic definitions and properties of $q$-difference calculus can be found in the book [4]. For the fractional $q$-difference calculus originating with work by Al-Salam [5] and Agarwal [6], we refer to the book of Annaby and Mansour [7]. Many intensive works on $q$-difference equations and fractional $q$-difference equations have been conducted (see [8-23]). However, the study of the boundary value problem of nonlinear $q$-difference equations is in deficiency. Examples of such scant works are as follows.

In 2015, Agarwal et al. [19] proposed the nonlinear $q$-integrodifference equation with non-separated boundary condition given by

$$
\left\{\begin{array}{l}
D_{q}^{2} u(t)=f(t, u(t))+I_{q} g(t, u(t)), \quad t \in I_{q}, \\
u(0)=\eta u(T), \quad D_{q} u(0)=\eta D_{q} u(T),
\end{array}\right.
$$

where $f, g \in C\left(I_{q} \times \mathbb{R}, \mathbb{R}\right), I_{q}=[0, T] \cap q^{\bar{N}}, q^{\bar{N}}:=\left\{q^{n} \mid n \in \mathbb{N}\right\} \cup\{0\}, T \in q^{\bar{N}}$, and $\eta \neq 1$. They presented sufficient conditions for the existence and nonexistence results of problem (1.1).

In [15], Ahmad et al. investigated the existence of solutions for the Caputo fractional $q$-difference integral equation with two different fractional orders and nonlocal boundary

(c) 2016 Patanarapeelert et al. This article is distributed under the terms of the Creative Commons Attribution 4.0 International License (http://creativecommons.org/licenses/by/4.0/), which permits unrestricted use, distribution, and reproduction in any medium, provided you give appropriate credit to the original author(s) and the source, provide a link to the Creative Commons license, and indicate if changes were made. 
conditions

$$
\left\{\begin{array}{l}
{ }^{\mathrm{c}} D_{q}^{\beta}\left({ }^{\mathrm{c}} D_{q}^{\gamma}+\lambda\right) x(t)=p f(t, x(t))+k I_{q}^{\xi} g(t, x(t)), \quad t \in[0,1), \\
\alpha_{1} x(0)-\beta_{1}\left(t^{(1-\gamma)} D_{q} x(0)\right)_{t=0}=\sigma_{1} x\left(\eta_{1}\right), \\
\alpha_{2} x(1)-\beta_{2} D_{q}(1)=\sigma_{2} x\left(\eta_{2}\right)
\end{array}\right.
$$

where $\beta, \gamma, \xi \in(0,1), f, g$ are given continuous functions, $\lambda, p, k$ are real constants and $\alpha_{i}, \beta_{i}, \sigma_{i} \in \mathbb{R}, \eta_{i} \in(0,1), i=1,2$.

Recently, Sitthiwirattham [20] discussed the existence results of solutions to a fractional $q$-difference equation and a fractional $q$-integrodifference equation,

$$
\begin{aligned}
& D_{q}^{\alpha} x(t)=f\left(t, x(t), D_{w}^{v} x(t)\right), \\
& D_{q}^{\alpha} x(t)=f\left(t, x(t), \Psi_{w}^{\gamma} x(t)\right), \quad t \in[0, T],
\end{aligned}
$$

with nonlocal three-point fractional $p$-integral boundary conditions of the form

$$
\left\{\begin{array}{l}
x(\eta)=\rho(x), \\
I_{p}^{\beta} g(T) x(T)=\frac{1}{\Gamma_{p}(\beta)} \int_{0}^{T} g(s)(T-p s)^{(\beta-1)} x(s) d_{p} s=0,
\end{array}\right.
$$

where $p, q, w \in(0,1), \alpha \in(1,2], v \in(0,1], \beta, \gamma>0$, and $\eta \in(0, T)$ are given constants, $f \in$ $C([0, T] \times \mathbb{R} \times \mathbb{R}, \mathbb{R}), g \in C\left([0, T], \mathbb{R}^{+}\right)$are given functions, and $\rho \in C([0, T], \mathbb{R}) \rightarrow \mathbb{R}$ is a given functional. For $\varphi \in C([0, T] \times[0, T],[0, \infty))$, define $\Psi_{w}^{\gamma} x(t):=\left(I_{w}^{\gamma} \varphi x\right)(t)=\frac{1}{\Gamma_{w}(\gamma)} \int_{0}^{t}(t-$ $w s)^{(\gamma-1)} \varphi(t, s) x(s) d_{w} s$.

To gain further insight in nonlinear $q$-integrodifference equations, in this paper, we devote our attention to the study of the existence and uniqueness for a sequential $q$-integrodifference boundary value problem involving two different fractional orders and six different numbers of $q$ in derivatives and integrals of the form

$$
\left\{\begin{array}{l}
D_{q}\left[\rho(t) D_{p}^{\gamma}\left(\kappa+D_{o}\right)\right] x(t)=f\left(t, x(t), D_{w}\left[e_{o}^{\kappa t} x(t)\right], \Psi_{\nu} x(t)\right), \\
x(0)=x(T), \\
\left(D_{o}\left[e_{o}^{\kappa t} x(t)\right]\right)_{t=0}=D_{o}\left[e_{o}^{\kappa T} x(T)\right], \\
I_{r}^{\theta} \sigma(T) x(T)=0,
\end{array}\right.
$$

where $t \in I_{\alpha}^{T}:=\left\{\alpha^{k} T: k \in \mathbb{N}\right\} \cup\{0, T\} ; \gamma, \theta \in(0,1], p=\frac{p_{1}}{p_{2}}, q=\frac{q_{1}}{q_{2}}, o=\frac{o_{1}}{o_{2}}, r=\frac{r_{1}}{r_{2}}, w=\frac{w_{1}}{w_{2}}, v=$ $\frac{v_{1}}{v_{2}}$, and $\alpha=\frac{1}{\operatorname{LCM}\left(p_{2}, q_{2}, o_{2}, r_{2}, w_{2}, v_{2}\right)}$ are proper fractions with $w \leq o$, LCM is the least common multiple; $\kappa \leq \frac{1}{T} ; \rho, \sigma \in C\left(I_{\alpha}^{T}, \mathbb{R}^{+}\right)$and $f \in C\left(I_{\alpha}^{T} \times \mathbb{R} \times \mathbb{R} \times \mathbb{R}, \mathbb{R}\right)$ are given functions; and for $\varphi \in C\left(I_{\alpha}^{T} \times I_{\alpha}^{T},[0, \infty)\right)$, define $\Psi_{\nu} x(t):=\left(I_{\nu} \varphi x\right)(t)=\int_{0}^{t} \varphi(t, s) x(s) d_{\nu} s$.

From our problem, we see that there are six different values of the $q$ numbers consisting of $q, p, o, w$-derivatives and $v, r$-integrals. This paper is organized as follows. We give some basis definitions, some properties of the $q$-difference operator and lemma as material used for this study in Section 2. To achieve proving the existence and uniqueness of solution of the given problem (1.5), we employ Banach's contraction mapping principle and Krasnoselskii's fixed point theorem in Section 3. Using our main results, we provide an example in Section 4. 


\section{Preliminaries}

In the following, there are notations, definitions, and lemmas which are used in the main results. Let $q \in(0,1)$ and define

$$
[n]_{q}:=\frac{1-q^{n}}{1-q}=q^{n-1}+\cdots+q+1 \quad \text { and } \quad[n]_{q} !:=\prod_{k=1}^{n} \frac{1-q^{k}}{1-q}, \quad n \in \mathbb{R} .
$$

The $q$-analog of the exponential function is

$$
e_{q}^{x}:=\sum_{k=0}^{\infty} \frac{x^{k}}{[k]_{q} !}
$$

The $q$-analog of the power function $(a-b)^{(n)}$ with $n \in \mathbb{N}_{0}:=[0,1,2, \ldots]$ is

$$
(a-b)^{(0)}:=1, \quad(a-b)^{(n)}:=\prod_{k=0}^{n-1}\left(a-b q^{k}\right), \quad a, b \in \mathbb{R} .
$$

More generally, if $\alpha \in \mathbb{R}$, then

$$
(a-b)^{(\alpha)}:=a^{\alpha} \prod_{n=0}^{\infty} \frac{1-\left(\frac{b}{a}\right) q^{n}}{1-\left(\frac{b}{a}\right) q^{\alpha+n}}, \quad a \neq 0 .
$$

Note that if $b=0$ then $a^{(\alpha)}=a^{\alpha}$. We also use the notation $0^{(\alpha)}=0$ for $\alpha>0$. The $q$-gamma function is defined by

$$
\Gamma_{q}(x):=\frac{(1-q)^{(x-1)}}{(1-q)^{x-1}}, \quad x \in \mathbb{R} \backslash\{0,-1,-2, \ldots\},
$$

and satisfies $\Gamma_{q}(x+1)=[x]_{q} \Gamma_{q}(x)$.

For any $x, s>0$, the $q$-beta function is defined by

$$
\begin{aligned}
B_{q}(x, s) & :=\int_{0}^{1} t^{(x-1)}(1-q t)^{(s-1)} d_{q} t \\
& =(1-q) \sum_{n=0}^{\infty} q^{n}\left(1-q^{n+1}\right)^{(\alpha-1)}\left(q^{n}\right)^{(x-1)}=\frac{\Gamma_{q}(x) \Gamma_{q}(s)}{\Gamma_{q}(x+s)} .
\end{aligned}
$$

Definition 2.1 [6] For $q \in(0,1)$, the $q$-derivative of a real function $f$ is defined by

$$
D_{q} f(t)=\frac{f(t)-f(q t)}{(1-q) t} \quad \text { and } \quad D_{q} f(0)=\lim _{t \rightarrow 0} D_{q} f(t) .
$$

For higher order $q$-derivatives of $f$, we define

$$
D_{q}^{n} f(t)=D_{q} D_{q}^{n-1} f(t), \quad n \in \mathbb{N} \quad \text { and } \quad D_{q}^{0} f(t)=f(t) .
$$

Next, if $f$ is a function defined on the interval $[0, T], q$-integral is defined as

$$
I_{q} f(t)=\int_{0}^{t} f(s) d_{q} s=\sum_{n=0}^{\infty} t(1-q) q^{n} f\left(t q^{n}\right) .
$$


Note from the last term of the above definition that the infinite series is convergent.

Definition 2.2 [6] For $\alpha \geq 0$ and $f$ defined on $[0, T]$, the fractional $q$-integral of the Riemann-Liouville type is defined by

$$
\begin{aligned}
\left(I_{q}^{\alpha} f\right)(x) & :=\frac{1}{\Gamma_{q}(\alpha)} \int_{0}^{x}(x-q t)^{(\alpha-1)} f(t) d_{q} t \\
& =\frac{x(1-q)}{\Gamma_{q}(\alpha)} \sum_{n=0}^{\infty} q^{n}\left(x-x^{n+1}\right)^{(\alpha-1)} f\left(x q^{n}\right) \\
& =\frac{x^{\alpha}(1-q)}{\Gamma_{q}(\alpha)} \sum_{n=0}^{\infty} q^{n}\left(1-q^{n+1}\right)^{(\alpha-1)} f\left(x q^{n}\right)
\end{aligned}
$$

and $\left(I_{q}^{0} f\right)(x)=f(x)$.

Definition 2.3 [8] For $\alpha \geq 0$ and $f$ defined on $[0, T]$, the fractional $q$-derivative of the Riemann-Liouville type of order $\alpha$ is defined by

$$
\left(D_{q}^{\alpha} f\right)(x):=\left(D_{q}^{m} I_{q}^{m-\alpha} f\right)(x), \quad \alpha>0 \quad \text { and } \quad\left(D_{q}^{0} f\right)(x)=f(x)
$$

where $m$ is the smallest integer that is greater than or equal to $\alpha$.

Lemma 2.1 [6] Let $\alpha, \beta \geq 0$ and $f$ be a function defined on $[0, T]$. Then the next formulas hold:

(i) $\left(I_{q}^{\beta} I_{q}^{\alpha} f\right)(x)=\left(I_{q}^{\alpha+\beta} f\right)(x)$,

(ii) $\left(D_{q}^{\alpha} I_{q}^{\alpha} f\right)(x)=f(x)$.

Lemma 2.2 [8] Let $\alpha>0$ and $N$ be a positive integer. Then the following equality holds:

$$
\left(I_{q}^{\alpha} D_{q}^{N} f\right)(x)=\left(D_{q}^{N} I_{q}^{\alpha} f\right)(x)-\sum_{k=0}^{N-1} \frac{x^{\alpha-N+k}}{\Gamma_{q}(\alpha+k-N+1)}\left(D_{q}^{k} f\right)(0) .
$$

Lemma 2.3 [17] Let $\alpha, \beta \geq 0$ and $0<p, q<1$. Then the following formulas hold:

$$
\begin{aligned}
& \text { (i) } \int_{0}^{\eta}(\eta-q t)^{(\alpha-1)} t^{(\beta)} d_{q} t=\eta^{\alpha+\beta} B_{q}(\alpha, \beta+1), \\
& \text { (ii) } \int_{0}^{\eta} \int_{0}^{s}(\eta-p s)^{(\alpha-1)}(s-q t)^{(\beta-1)} d_{q} t d_{p} s=\frac{\eta^{\alpha+\beta}}{[\beta]_{q}} B_{p}(\alpha, \beta+1) .
\end{aligned}
$$

To define the solution of the boundary value problem (1.5), we need the following lemma, which deals with a linear variant of the boundary value problem (1.5) and gives a representation of the solution.

Lemma 2.4 Let $p=\frac{p_{1}}{p_{2}}, q=\frac{q_{1}}{q_{2}}, o=\frac{o_{1}}{o_{2}}, r=\frac{r_{1}}{r_{2}}$, and $\beta=\frac{1}{\operatorname{LCM}\left(p_{2}, q_{2}, o_{2}, r_{2}\right)}$ be proper fractions, $\kappa>0$. For $h \in C\left(I_{\beta}^{T}, \mathbb{R}\right)$ and $\rho, \sigma \in C\left(I_{\beta}^{T}, \mathbb{R}^{+}\right)$, the solution for the problem

$$
D_{q}\left[\rho(t) D_{p}^{\gamma}\left(\kappa+D_{o}\right)\right] x(t)=h(t), \quad t \in I_{\beta}^{T},
$$




$$
\left\{\begin{array}{l}
x(0)=x(T) \\
\left(D_{o}\left[e_{o}^{\kappa t} x(t)\right]\right)_{t=0}=D_{o}\left[e_{o}^{\kappa T} x(T)\right] \\
I_{r}^{\theta} \sigma(T) x(T)=0
\end{array}\right.
$$

is of the form

$$
\begin{aligned}
e_{o}^{\kappa t} x(t)= & \frac{1}{\Gamma_{p}(\gamma)} \int_{0}^{t} \int_{0}^{s} \int_{0}^{z} \frac{e_{o}^{\kappa s}(s-p z)^{(\gamma-1)}}{\rho(z)} h(u) d_{q} u d_{p} z d_{o} s-\mathbf{O}_{1}(h) \\
& -\mathbf{O}_{2}(h)\left[\frac{1}{\Gamma_{p}(\gamma)} \int_{0}^{t} \int_{0}^{s} \frac{e_{o}^{\kappa s}(s-p z)^{(\gamma-1)}}{\rho(z)} d_{p} z d_{o} s-\mathbf{A}\right]+\mathbf{O}_{3}(h)\left[e_{o}^{\kappa t}+\mathbf{B}\right],
\end{aligned}
$$

where the functionals $\mathbf{O}_{i}(h), i=1,2,3$ are defined by

$$
\begin{aligned}
\mathbf{O}_{1}(h)= & \frac{1}{\Gamma_{p}(\gamma) \Gamma_{r}(\theta) \int_{0}^{T} \sigma(s)(T-r s)^{(\theta-1)} d_{r} s} \\
& \times \int_{0}^{T} \int_{0}^{s} \int_{0}^{z} \int_{0}^{y} \frac{\sigma(s) e_{o}^{\kappa z}(T-r s)^{(\theta-1)}(z-p y)^{(\gamma-1)}}{e_{o}^{\kappa T} \rho(y)} h(u) d_{q} u d_{p} y d_{o} z d_{r} s, \\
\mathbf{O}_{2}(h)= & \frac{e_{o}^{\kappa T}}{\Gamma_{p}(\gamma) \int_{0}^{T} \frac{(T-p s)^{(\gamma-1)}}{\rho(s)} d_{p} s} \int_{0}^{T} \int_{0}^{s} \frac{(T-p s)^{(\gamma-1)}}{\rho(s)} h(u) d_{q} u d_{p} s, \\
\mathbf{O}_{3}(h)= & \frac{1}{\Gamma_{p}(\gamma)\left(e_{o}^{\kappa T}-1\right)}\left(\int_{0}^{T} \int_{0}^{s} \int_{0}^{z} e_{o}^{\kappa s} \frac{(s-p z)^{(\gamma-1)}}{\rho(z)} h(u) d_{q} u d_{p} z d_{o} s\right. \\
& \left.+\frac{e_{o}^{\kappa T} \int_{0}^{T} \int_{0}^{s} e_{o}^{\kappa s} \frac{(T-p z)^{(\gamma-1)}}{\rho(z)} d_{p} z d_{o} s}{\int_{0}^{T} \frac{(T-p s)^{(\gamma-1)}}{\rho(s)} d_{p} s} \int_{0}^{s} \frac{(T-p s)^{(\gamma-1)}}{\rho(s)} h(u) d_{q} u d_{p} s\right),
\end{aligned}
$$

and the constants

$$
\begin{aligned}
\mathbf{A}= & \frac{1}{\Gamma_{p}(\gamma) \int_{0}^{T} \sigma(s)(T-r s)^{(\beta-1)} d_{r} s} \\
& \times \int_{0}^{T} \int_{0}^{s} \int_{0}^{z} \frac{\sigma(s) e_{o}^{\kappa z}(T-r s)^{(\beta-1)}(z-p y)^{(\gamma-1)}}{e_{o}^{\kappa T} \rho(y)} d_{p} y d_{o} z d_{r} s, \\
\mathbf{B}= & \frac{1}{\int_{0}^{T} \sigma(s)(T-r s)^{(\theta-1)} d_{r} s} \int_{0}^{T} \int_{0}^{s} \sigma(s) e_{o}^{\kappa z}(T-r s)^{(\theta-1)} d_{o} z d_{r} s .
\end{aligned}
$$

Proof We first $q$-integrate (2.1) to obtain

$$
D_{p}^{\gamma}\left(\kappa+D_{o}\right) x(t)=\frac{1}{\rho(t)}\left(\int_{0}^{t} h(s) d_{q} s+C_{1}\right) .
$$

Then, taking the $p$-integral of order $\gamma$ for (2.9), we have

$$
\begin{aligned}
\left(\kappa+D_{o}\right) x(t)= & \frac{1}{\Gamma_{p}(\gamma)} \int_{0}^{t} \int_{0}^{s} \frac{(t-p s)^{(\gamma-1)} h(u)}{\rho(s)} d_{q} u d_{p} s \\
& +\frac{C_{1}}{\Gamma_{p}(\gamma)} \int_{0}^{t} \frac{(t-p s)^{(\gamma-1)}}{\rho(s)} d_{p} s+C_{2},
\end{aligned}
$$


which can alternatively be written as

$$
\begin{aligned}
D_{o}\left(e_{o}^{\kappa t} x(t)\right)= & \left(\kappa e_{o}^{\kappa t}+e_{o}^{\kappa t} D_{o}\right) x(t) \\
= & \frac{e_{o}^{\kappa t}}{\Gamma_{p}(\gamma)} \int_{0}^{t} \int_{0}^{s} \frac{(t-p s)^{(\gamma-1)} h(u)}{\rho(s)} d_{q} u d_{p} s \\
& +\frac{C_{1} e_{o}^{\kappa t}}{\Gamma_{p}(\gamma)} \int_{0}^{t} \frac{(t-p s)^{(\gamma-1)}}{\rho(s)} d_{p} s+C_{2} e_{o}^{\kappa t} .
\end{aligned}
$$

Taking the $o$-integration of (2.11), we have

$$
\begin{aligned}
e_{o}^{\kappa t} x(t)= & \frac{1}{\Gamma_{p}(\gamma)} \int_{0}^{t} \int_{0}^{s} \int_{0}^{z} e_{o}^{\kappa s} \frac{(s-p z)^{(\gamma-1)} h(u)}{\rho(z)} d_{q} u d_{p} z d_{o} s \\
& +\frac{C_{1}}{\Gamma_{p}(\gamma)} \int_{0}^{t} \int_{0}^{s} e_{o}^{\kappa s} \frac{(s-p z)^{(\gamma-1)}}{\rho(z)} d_{p} z d_{o} s+C_{2} \int_{0}^{t} e_{o}^{\kappa s} d_{o} s+C_{3} .
\end{aligned}
$$

Letting $t=0, T$ in (2.11) and (2.12), and employing the first and second conditions of (2.2), we get

$$
\begin{aligned}
C_{1}= & -\frac{e_{o}^{\kappa T} \int_{0}^{T} \int_{0}^{s} \frac{(T-p s(\gamma-1)}{\rho(s)} h(u) d_{q} u d_{p} s}{\int_{0}^{T} \frac{(T-p s)(\gamma-1)}{\rho(s)} d_{p} s}, \\
C_{2}= & \frac{1}{\Gamma_{p}(\gamma) \int_{0}^{T} e_{o}^{\kappa s} d_{o} s}\left(\int_{0}^{T} \int_{0}^{s} \int_{0}^{z} e_{o}^{\kappa s} \frac{(s-p z)^{(\gamma-1)}}{\rho(z)} h(u) d_{q} u d_{p} z d_{o} s\right. \\
& \left.+\frac{e_{o}^{\kappa T} \int_{0}^{T} \int_{0}^{s} \frac{(T-p s)(\gamma-1)}{\rho(s)} h(u) d_{q} u d_{p} s}{\int_{0}^{T} \frac{(T-p s)(\gamma-1)}{\rho(s)} d_{p} s} \int_{0}^{T} \int_{0}^{s} e_{o}^{\kappa s} \frac{(T-p z)^{(\gamma-1)}}{\rho(z)} d_{p} z d_{o} s\right) .
\end{aligned}
$$

Next, taking the $r$-integral of order $\theta$ for $\sigma(t) x(t)$ where $t \in I_{\beta}^{T}$, we have

$$
\begin{aligned}
I_{r}^{\theta} \sigma(t) x(t)= & \frac{1}{\Gamma_{p}(\gamma) \Gamma_{r}(\theta)} \int_{0}^{t} \int_{0}^{s} \int_{0}^{z} \int_{0}^{y} \frac{\sigma(s) e_{o}^{\kappa z}(t-r s)^{(\theta-1)}(z-p y)^{(\gamma-1)}}{e_{o}^{\kappa t} \rho(y)} \\
& \times h(u) d_{q} u d_{p} y d_{o} z d_{r} s \\
& +\frac{C_{1}}{\Gamma_{p}(\gamma) \Gamma_{r}(\theta)} \int_{0}^{t} \int_{0}^{s} \int_{0}^{z} \frac{\sigma(s) e_{o}^{\kappa z}(t-r s)^{(\theta-1)}(z-p y)^{(\gamma-1)}}{e_{o}^{\kappa t} \rho(y)} d_{p} y d_{o} z d_{r} s \\
& +\frac{C_{2}}{\Gamma_{r}(\theta)} \int_{0}^{t} \int_{0}^{s} \sigma(s) e_{o}^{\kappa z}(t-r s)^{(\theta-1)} d_{p} y d_{o} z d_{r} s \\
& +\frac{C_{3}}{\Gamma_{r}(\theta)} \int_{0}^{t} \sigma(s)(t-r s)^{(\theta-1)} d_{r} s .
\end{aligned}
$$

By substituting $C_{1}, C_{2}$ into (2.10) and employing the third condition of (2.2), we find that

$$
\begin{aligned}
C_{3}= & \frac{1}{\Gamma_{p}(\gamma) \Gamma_{r}(\theta) \int_{0}^{T} \sigma(s)(T-r s)^{(\beta-1)} d_{r} s}\left\{\frac{e_{o}^{\kappa T} \int_{0}^{T} \int_{0}^{s} \frac{(T-p s)(\gamma-1)}{\rho(s)} h(u) d_{q} u d_{p} s}{\int_{0}^{T} \frac{(T-p s)^{(\gamma-1)}}{\rho(s)} d_{p} s}\right. \\
& \times \int_{0}^{T} \int_{0}^{s} \int_{0}^{z} \frac{\sigma(s) e_{o}^{\kappa z}(T-r s)^{(\beta-1)}(z-p y)^{(\gamma-1)}}{e_{o}^{\kappa T} \rho(y)} h(u) d_{p} y d_{o} z d_{r} s
\end{aligned}
$$




$$
\begin{aligned}
& -\frac{1}{\int_{0}^{T} e_{o}^{\kappa s} d_{o} s}\left(\int_{0}^{T} \int_{0}^{s} \int_{0}^{z} e_{o}^{\kappa s} \frac{(s-p z)^{(\gamma-1)}}{\rho(z)} h(u) d_{q} u d_{p} z d_{o} s\right. \\
& \left.+\frac{e_{o}^{\kappa T} \int_{0}^{T} \int_{0}^{s} \frac{(T-p s)^{(\gamma-1)}}{\rho(s)} h(u) d_{q} u d_{p} s}{\int_{0}^{T} \frac{\left(T-p s()^{(\gamma-1)}\right.}{\rho(s)} d_{p} s} \int_{0}^{T} \int_{0}^{s} e_{o}^{\kappa s} \frac{(T-p z)^{(\gamma-1)}}{\rho(z)} d_{p} z d_{o} s\right) \\
& \times \int_{0}^{T} \int_{0}^{s} \sigma(s) e_{o}^{\kappa z}(T-r s)^{(\beta-1)} d_{p} y d_{o} z d_{r} s \\
& \left.-\int_{0}^{T} \int_{0}^{s} \int_{0}^{z} \int_{0}^{y} \frac{\sigma(s) e_{o}^{\kappa z}(T-r s)^{(\beta-1)}(z-p y)^{(\gamma-1)}}{e_{o}^{\kappa T} \rho(y)} h(u) d_{q} u d_{p} y d_{o} z d_{r} s\right\} .
\end{aligned}
$$

Substituting $C_{1}, C_{2}$, and $C_{3}$ into (2.12), we obtain (2.3).

On the other hand, we shall show that (2.3) is the solution of problem (2.1)-(2.2). First of all, taking the $o$-derivative for (2.3), we obtain

$$
\begin{aligned}
D_{o}\left(e_{o}^{\kappa t} x(t)\right)= & \frac{e_{o}^{\kappa t}}{\Gamma_{p}(\gamma)} \int_{0}^{t} \int_{0}^{s} \frac{(t-p s)^{(\gamma-1)} h(u)}{\rho(s)} d_{q} u d_{p} s \\
& -\frac{e_{o}^{\kappa t} \mathbf{O}_{2}(h)}{\Gamma_{p}(\gamma)} \int_{0}^{t} \frac{(t-p s)^{(\gamma-1)}}{\rho(s)} d_{p} s+\mathbf{O}_{3}(h) \kappa e_{o}^{\kappa t},
\end{aligned}
$$

which can alternatively be written as

$$
\begin{aligned}
\left(\kappa+D_{o}\right) x(t)= & \frac{1}{\Gamma_{p}(\gamma)} \int_{0}^{t} \int_{0}^{s} \frac{(t-p s)^{(\gamma-1)} h(u)}{\rho(s)} d_{q} u d_{p} s \\
& -\frac{\mathbf{O}_{2}(h)}{\Gamma_{p}(\gamma)} \int_{0}^{t} \frac{(t-p s)^{(\gamma-1)}}{\rho(s)} d_{p} s+\mathbf{O}_{3}(h) .
\end{aligned}
$$

Taking the $p$-derivative of order $\gamma$ for (2.18), we have

$$
\rho(t) D_{p}^{\gamma}\left(\kappa+D_{o}\right) x(t)=\int_{0}^{t} h(s) d_{q} s+\mathbf{O}_{2}(h)
$$

Finally, taking the $q$-derivative for (2.19), we obtain (2.1). The proof is completed.

\section{Main results}

In order to prove the main results, we need to transform the boundary value problem (1.5) into a fixed point problem. We employ Lemma 2.4 by letting $P(t)=\frac{1}{\rho(t)}$, the Banach space $\mathcal{C}=C\left(I_{\alpha}^{T}, \mathbb{R}\right)=\left\{x: I_{\alpha}^{T} \rightarrow \mathbb{R} \mid x \in C\left(I_{\alpha}^{T}\right)\right\}$ equipped with a topology of uniform convergence with respect to the norm

$$
\|x\|_{\mathcal{C}}=\|x\|+\left\|D_{w}\left[e_{o}^{\kappa t} x(t)\right]\right\|
$$

where $\|x\|=\sup _{t \in I_{\alpha}^{T}}|x(t)|$ and $\left\|D_{w}\left[e_{o}^{\kappa t} x(t)\right]\right\|=\sup _{t \in I_{\alpha}^{T}}\left|D_{w}\left[e_{o}^{\kappa t} x(t)\right]\right|$. Define an operator $F$ : $\mathcal{C} \rightarrow \mathcal{C}$ by

$$
\begin{aligned}
(F x)(t)= & \frac{e_{o}^{-\kappa t}}{\Gamma_{p}(\gamma)} \int_{0}^{t} \int_{0}^{s} \int_{0}^{z} e_{o}^{\kappa s}(s-p z)^{(\gamma-1)} P(z) \\
& \times f\left(u, x(u), D_{w}\left[e_{o}^{\kappa u} x(u)\right], \Psi_{\nu} x(u)\right) d_{q} u d_{p} z d_{o} s
\end{aligned}
$$




$$
\begin{aligned}
& -e_{o}^{-\kappa t} \mathbf{O}_{1}^{*}(f)-e_{o}^{-\kappa t} \mathbf{O}_{2}^{*}(f)\left[\frac{1}{\Gamma_{p}(\gamma)} \int_{0}^{t} \int_{0}^{s} e_{o}^{\kappa s}(s-p z)^{(\gamma-1)} P(z) d_{p} z d_{o} s-\mathbf{A}\right] \\
& +e_{o}^{-\kappa t} \mathbf{O}_{3}^{*}(f)\left[\int_{0}^{t} e_{o}^{\kappa s} d_{o} s-\mathbf{B}\right],
\end{aligned}
$$

where $p=\frac{p_{1}}{p_{2}}, q=\frac{q_{1}}{q_{2}}, o=\frac{o_{1}}{o_{2}}, r=\frac{r_{1}}{r_{2}}, w=\frac{w_{1}}{w_{2}}, v=\frac{v_{1}}{v_{2}}, \alpha=\frac{1}{\operatorname{LCM}\left(p_{2}, q_{2}, o_{2}, r_{2}, w_{2}, v_{2}\right)}$ are proper fractions, $w \leq o ; \kappa \leq \frac{1}{T}$; and $\mathbf{O}_{i}^{*}(f), i=1,2,3$, are defined by

$$
\begin{aligned}
\mathbf{O}_{1}^{*}(f)= & \frac{1}{\Gamma_{p}(\gamma) \int_{0}^{T} \sigma(s)(T-r s)^{(\theta-1)} d_{r} s} \int_{0}^{T} \int_{0}^{s} \int_{0}^{z} \int_{0}^{y} \sigma(s) e_{o}^{\kappa(z-T)}(T-r s)^{(\theta-1)} \\
& \times(z-p y)^{(\gamma-1)} P(y) f\left(u, x(u), D_{w}\left[e_{o}^{\kappa u} x(u)\right], \Psi_{\nu} x(u)\right) d_{q} u d_{p} y d_{o} z d_{r} s \\
\mathbf{O}_{2}^{*}(f)= & \frac{e_{o}^{\kappa T}}{\int_{0}^{T}(T-p s)^{(\gamma-1)} P(s) d_{p} s} \int_{0}^{T} \int_{0}^{s}(T-p s)^{(\gamma-1)} P(s) \\
& \times f\left(u, x(u), D_{w}\left[e_{o}^{\kappa u} x(u)\right], \Psi_{\nu} x(u)\right) d_{q} u d_{p} s, \\
\mathbf{O}_{3}^{*}(f)= & \frac{1}{\Gamma_{p}(\gamma)\left[e_{o}^{\kappa T}-1\right]} \\
& \times\left\{\int_{0}^{T} \int_{0}^{s} \int_{0}^{z} e_{o}^{\kappa s}(s-p z)^{(\gamma-1)} P(z) f\left(u, x(u), D_{w}\left[e_{o}^{\kappa u} x(u)\right], \Psi_{\nu} x(u)\right) d_{q} u d_{p} z d_{o} s\right. \\
& +\frac{e_{o}^{\kappa T} \int_{0}^{T} \int_{0}^{s} e_{o}^{\kappa s}(T-p z)^{(\gamma-1)} P(z) d_{p} z d_{o} s}{\int_{0}^{T}(T-p s)^{(\gamma-1)} P(s) d_{p} s} \\
& \left.\times \int_{0}^{T} \int_{0}^{s}(T-p s)^{(\gamma-1)} P(s) f\left(u, x(u), D_{w}\left[e_{o}^{\kappa u} x(u)\right], \Psi_{\nu} x(u)\right) d_{q} u d_{p} s\right\}
\end{aligned}
$$

with the constants A, B defined as (2.7)-(2.8), respectively.

Clearly problem (1.5) has solutions if and only if the operator $F$ has fixed points.

Theorem 3.1 Assume $\sigma, P: I_{\alpha}^{T} \rightarrow \mathbb{R}^{+}, f: I_{\alpha}^{T} \times \mathbb{R} \times \mathbb{R} \times \mathbb{R} \rightarrow \mathbb{R}$ and $\varphi: I_{\alpha}^{T} \times I_{\alpha}^{T} \rightarrow[0, \infty)$ are continuous, let $\varphi_{0}:=\sup _{(t, s) \in I_{\alpha}^{T} \times I_{\alpha}^{T}}\{\varphi(t, s)\}$. In addition, assume that $f, \sigma$, and $P$ satisfy the following conditions:

$\left(\mathrm{H}_{1}\right)$ there exist positive constants $L_{1}, L_{2}$, and $L_{3}$ such that

$$
\begin{aligned}
& \left|f\left(t, x, D_{w}\left(e_{o}^{\kappa t} x\right), \Psi_{\vee} x\right)-f\left(t, y, D_{w}\left(e_{o}^{\kappa t} y\right), \Psi_{v} y\right)\right| \\
& \quad \leq L_{1}|x-y|+L_{2}\left|D_{w}\left(e_{o}^{\kappa t} x\right)-D_{w}\left(e_{o}^{\kappa t} y\right)\right|+L_{3}\left|\Psi_{\nu} x-\Psi_{\nu} y\right|,
\end{aligned}
$$

for all $t \in I_{\alpha}^{T}$ and $x, y \in \mathbb{R}$,

$\left(\mathrm{H}_{2}\right) \quad 0<m<\sigma(t)<M$ and $0<n<P(t)<N$, for all $t \in I_{\alpha}^{T}$,

$\left(\mathrm{H}_{3}\right) \lambda\left(\Omega_{1}+\Omega_{2}\right)<1$,

where

$$
\begin{aligned}
& \lambda=\max \left\{L_{1}+\varphi_{0} T L_{3}, L_{2}\right\}, \\
& \Omega_{1}=\frac{T^{\gamma+2} N}{\Gamma_{p}(\gamma+2)[\gamma+2]_{o}}\left[1+M[\theta]_{r} B_{r}(\theta, \gamma+3)\right]+\left[\frac{T}{[\gamma+1]_{o}}+\frac{M[\theta]_{r} B_{r}(\theta, \gamma+2)}{m[r+1]_{p}}\right]
\end{aligned}
$$




$$
\begin{aligned}
& \times \frac{N^{2} e_{o}^{\kappa T} T^{\gamma}}{n \Gamma_{p}(\gamma+2)}+\frac{N e_{o}^{\kappa T} T^{\gamma+1}}{n \Gamma_{p}(\gamma+2)}\left[\frac{T}{[\gamma+2]_{o}}+\frac{1}{[\gamma+1]_{o}}\right]\left[1+\frac{M e_{o}^{\kappa T}}{m[\theta+1]_{r}}\right], \\
\Omega_{2}= & \frac{T^{\gamma} N[\gamma+1]_{w}}{\Gamma_{p}(\gamma+2)}\left[\frac{T}{[\gamma+2]_{o}}++\frac{N e_{o}^{\kappa T}}{n[\gamma+1]_{o}}\right] \\
& +\frac{N \kappa\left(e_{o}^{\kappa T}\right)^{2} T^{\gamma}}{\Gamma_{p}(\gamma+2)}\left[\frac{T}{[\gamma+2]_{o}}+\frac{1}{[\gamma+1]_{o}}\right] .
\end{aligned}
$$

Then the given boundary value problem (1.5) has a unique solution.

Proof As mentioned earlier, we need to transform the boundary value problem (1.5) into a fixed point problem $x=F x$, where $F: \mathcal{C} \rightarrow \mathcal{C}$ is defined by (3.1). We assume that $\sup _{t \in I_{\alpha}^{T}}|f(t, 0,0,0)|=K$ and choose a constant $R$ satisfying the inequality

$$
R \geq \frac{K\left(\Omega_{1}+\Omega_{2}\right)}{1-\lambda\left(\Omega_{1}+\Omega_{2}\right)} .
$$

For $x \in B_{R}$, the following is to prove that $F B_{R} \subset B_{R}$, where $B_{R}=\{x \in \mathcal{C}:\|x\| \leq R\}$. First we consider

$$
\begin{aligned}
&\left|\mathbf{O}_{1}^{*}(f)\right| \leq \sup _{t \in I_{\alpha}^{T}} \mid \frac{M N}{m \Gamma_{p}(\gamma) \int_{0}^{T}(T-r s)^{(\theta-1)} d_{r} s} \int_{0}^{T} \int_{0}^{s} \int_{0}^{z} \int_{0}^{y}(T-r s)^{(\theta-1)}(z-p y)^{(\gamma-1)} \\
& \times\left(\left|f\left(u, x(u), D_{w}\left[e_{o}^{\kappa u} x(u)\right], \Psi_{\nu} x(u)\right)-f(u, 0,0,0)\right|\right. \\
&+|f(u, 0,0,0)|) d_{q} u d_{p} y d_{o} z d_{r} s \mid \\
& \leq \sup _{t \in I_{\alpha}^{T}} \mid \frac{M N\left(\left(L_{1}+\varphi_{0} T L_{3}\right)|x(u)|+L_{2}\left|D_{w}\left[e_{o}^{\kappa u} x(u)\right]\right|+K\right)}{m \Gamma_{p}(\gamma) \int_{0}^{T}(T-r s)^{(\theta-1)} d_{r} s} \\
& \times \int_{0}^{T} \int_{0}^{s} \int_{0}^{z} \int_{0}^{y}(T-r s)^{(\theta-1)}(z-p y)^{(\gamma-1)} d_{q} u d_{p} y d_{o} z d_{r} s \mid \\
& \leq \sup _{t \in I_{\alpha}^{T}}\left\{\left(\lambda\|x\|_{\mathcal{C}}+K\right)\left(\frac{M N \Gamma_{r}(\theta+1) \Gamma_{r}(\gamma+3) T^{\gamma+2}}{n \Gamma_{p}(\gamma+2) \Gamma_{r}(\theta+\gamma+3)[\gamma+2]_{o}}\right)\right\} \\
&\left|\mathbf{O}_{2}^{*}(f)\right| \leq \sup _{t \in I_{\alpha}^{T}} \mid \frac{N e_{o}^{\kappa T}}{n \int_{0}^{T}(T-p s)^{(\gamma-1)} d_{p} s} \int_{0}^{T} \int_{0}^{s}(T-p s)^{(\gamma-1)} \\
& \times\left(\left|f\left(u, x(u), D_{w}\left[e_{o}^{\kappa u} x(u)\right], \Psi_{\nu} x(u)\right)-f(u, 0,0,0)\right|\right. \\
&+|f(u, 0,0,0)|) d_{q} u d_{p} s \mid \\
&\left|\mathbf{O}_{3}^{*}(f)\right| \leq \sup _{t \in I_{\alpha}^{T}} \mid \frac{N e_{o}^{K T}}{\Gamma_{p}(\gamma)\left[e_{o}^{\kappa T}-1\right]}\left\{\int_{0}^{T} \int_{0}^{s} \int_{0}^{z}(s-p z)^{(\gamma-1)}\right. \\
& \leq \sup _{t \in I_{\alpha}^{T}} \mid \frac{N e_{o}^{\kappa T}\left(\left(L_{1}+\varphi_{0} T L_{3}\right)|x(u)|+L_{2}\left|D_{w}\left[e_{o}^{\kappa u} x(u)\right]\right|+K\right)}{n \int_{0}^{T}(T-p s)^{(\gamma-1)} d_{p} s} \\
& \times \int_{0}^{T} \int_{0}^{s}(T-p s)^{(\gamma-1)} d_{q} u d_{p} s \mid \\
&\qquad x \| \mathcal{C}+K)\left(\frac{N e_{o}^{\kappa T}}{n[\gamma+1]_{p}}\right),
\end{aligned}
$$




$$
\begin{aligned}
& \times\left(\left|f\left(u, x(u), D_{w}\left[e_{o}^{\kappa u} x(u)\right], \Psi_{\nu} x(u)\right)-f(u, 0,0,0)\right|\right. \\
& +|f(u, 0,0,0)|) d_{q} u d_{p} z d_{o} s \\
& +\frac{N e_{o}^{\kappa T} \int_{0}^{T} \int_{0}^{s}(T-p z)^{(\gamma-1)} d_{p} z d_{o} s}{n \int_{0}^{T}(T-p s)^{(\gamma-1)} d_{p} s} \int_{0}^{T} \int_{0}^{s}(T-p s)^{(\gamma-1)} \\
& \times\left(\left|f\left(u, x(u), D_{w}\left[e_{o}^{\kappa u} x(u)\right], \Psi_{\nu} x(u)\right)-f(u, 0,0,0)\right|\right. \\
& \left.+|f(u, 0,0,0)|) d_{q} u d_{p} s\right\} \mid \\
\leq & \sup _{t \in I_{\alpha}^{T}} \mid \frac{N e_{o}^{\kappa T}}{\Gamma_{p}(\gamma)\left[e_{o}^{\kappa T}-1\right]}\left(\left(L_{1}+\varphi_{0} T L_{3}\right)|x(u)|+L_{2}\left|D_{w}\left[e_{o}^{\kappa u} x(u)\right]\right|+K\right) \\
& \times\left\{\int_{0}^{T} \int_{0}^{s} \int_{0}^{z}(s-p z)^{(\gamma-1)} d_{q} u d_{p} z d_{o} s+\frac{N e_{o}^{\kappa T} \int_{0}^{T} \int_{0}^{s}(T-p z)^{(\gamma-1)} d_{p} z d_{o} s}{n \int_{0}^{T}(T-p s)^{(\gamma-1)} d_{p} s}\right. \\
& \left.\times \int_{0}^{T} \int_{0}^{s}(T-p s)^{(\gamma-1)} d_{q} u d_{p} s\right\} \mid \\
\leq & \left(\lambda\|x\|_{\mathcal{C}}+K\right)\left[\frac{N e_{o}^{\kappa T} T^{\gamma}}{n \Gamma_{p}(\gamma+2)}\left(\frac{T}{[\gamma+2]_{o}}+\frac{1}{[\gamma+1]_{o}}\right)\right],
\end{aligned}
$$

and

$$
\begin{aligned}
|\mathbf{A}| & \leq \frac{M N}{m \Gamma_{p}(\gamma) \int_{0}^{T}(T-r s)^{(\beta-1)} d_{r} s} \int_{0}^{T} \int_{0}^{s} \int_{0}^{z}(T-r s)^{(\beta-1)}(z-p y)^{(\gamma-1)} d_{p} y d_{o} z d_{r} s \\
& \leq \sup _{t \in I_{\alpha}^{T}}\left\{\frac{M N T^{\gamma+1} \Gamma_{r}(\gamma+2) \Gamma_{r}(\theta+1)}{m \Gamma_{p}(\gamma+2) \Gamma_{r}(\theta+\gamma+2)}\right\}, \\
|\mathbf{B}| & \leq \frac{M e_{o}^{\kappa T}}{m \int_{0}^{T}(T-r s)^{(\theta-1)} d_{r} s} \int_{0}^{T} \int_{0}^{s}(T-r s)^{(\theta-1)} d_{o} z d_{r} s \leq \frac{M T e_{o}^{\kappa T}}{m[\theta+1]_{r}} .
\end{aligned}
$$

Consequently, we have

$$
\begin{aligned}
|(F x)(t)| \leq & \sup _{t \in I_{\alpha}^{T}}\left\{\frac{N}{\Gamma_{p}(\gamma)}\left(\left(L_{1}+\varphi_{0} T L_{3}\right)|x(u)|+L_{2}\left|D_{w}\left[e_{o}^{\kappa u} x(u)\right]\right|+K\right)\right. \\
& \times \int_{0}^{t} \int_{0}^{s} \int_{0}^{z}(s-p z)^{(\gamma-1)} d_{q} u d_{p} z d_{o} s+e_{o}^{-\kappa t}\left|\mathbf{O}_{1}^{*}(f)\right|+\left|\mathbf{O}_{2}^{*}(f)\right| \\
& \left.\times\left[\frac{N}{\Gamma_{p}(\gamma)} \int_{0}^{t} \int_{0}^{s}(s-p z)^{(\gamma-1)} d_{p} z d_{o} s+e_{o}^{-\kappa t}|\mathbf{A}|\right]+\left|\mathbf{O}_{3}^{*}(f)\right|\left[1+e_{o}^{-\kappa t}|\mathbf{B}|\right]\right\} \\
\leq & \sup _{t \in I_{\alpha}^{T}}\left\{\frac{t^{\gamma+2} N\left(\lambda\|x\|_{\mathcal{C}}+K\right)}{\Gamma_{p}(\gamma+2)[\gamma+2]_{o}}+e_{o}^{-\kappa t}\left|\mathbf{O}_{1}^{*}(f)\right|\right. \\
& \left.+\left|\mathbf{O}_{2}^{*}(f)\right|\left[\frac{t^{\gamma+1} N}{\Gamma_{p}(\gamma+1)[\gamma+1]_{o}}+e_{o}^{-\kappa t}|\mathbf{A}|\right]+\left|\mathbf{O}_{3}^{*}(f)\right|\left[t+e_{o}^{-\kappa t}|\mathbf{B}|\right]\right\} \\
\leq & \left(\lambda\|x\|_{\mathcal{C}}+K\right)\left(\frac{T^{\gamma+2} N}{\Gamma_{p}(\gamma+2)[\gamma+2]_{o}}\left[1+M[\theta]_{r} B_{r}(\theta, \gamma+3)\right]\right. \\
& +\frac{N^{2} e_{o}^{\kappa T} T^{\gamma}}{n \Gamma_{p}(\gamma+2)}\left[\frac{T}{[\gamma+1]_{o}}+\frac{M[\theta]_{r} B_{r}(\theta, \gamma+2)}{m[r+1]_{p}}\right]
\end{aligned}
$$




$$
\begin{aligned}
& \left.+\frac{N e_{o}^{\kappa T} T^{\gamma+1}}{n \Gamma_{p}(\gamma+2)}\left[\frac{T}{[\gamma+2]_{o}}+\frac{1}{[\gamma+1]_{o}}\right]\left[1+\frac{M e_{o}^{\kappa T}}{m[\theta+1]_{r}}\right]\right) \\
= & \left(\lambda\|x\|_{\mathcal{C}}+K\right) \Omega_{1}
\end{aligned}
$$

and

$$
\begin{aligned}
& \left|D_{w}\left[e_{o}^{\kappa t}(F x)\right](t)\right| \\
& \leq \sup _{t \in I_{\alpha}^{T}}\left\{\frac{N}{\Gamma_{p}(\gamma)(1-w) t} \mid \int_{0}^{t} \int_{0}^{s} \int_{0}^{z} e_{o}^{\kappa s}(s-p z)^{(\gamma-1)}\right. \\
& \times f\left(u, x(u), D_{w}\left[e_{o}^{\kappa u} x(u)\right], \Psi_{v} x(u)\right) d_{q} u d_{p} z d_{o} s \\
& -\int_{0}^{w t} \int_{0}^{s} \int_{0}^{z} e_{o}^{\kappa s}(s-p z)^{(\gamma-1)} f\left(u, x(u), D_{w}\left[e_{o}^{\kappa u} x(u)\right], \Psi_{\nu} x(u)\right) d_{q} u d_{p} z d_{o} s \\
& +\frac{N\left|\mathbf{O}_{2}^{*}(f)\right|}{\Gamma_{p}(\gamma)(1-w) t}\left|\int_{0}^{t} \int_{0}^{s} e_{o}^{\kappa s}(s-p z)^{(\gamma-1)} d_{p} z d_{o} s-\int_{0}^{w t} \int_{0}^{s} e_{o}^{\kappa s}(s-p z)^{(\gamma-1)} d_{p} z d_{o} s\right| \\
& \left.+\left|\mathbf{O}_{3}^{*}(f)\right| \frac{\left|e_{o}^{\kappa t}-e_{o}^{\kappa w t}\right|}{(1-w) t}\right\} \\
& \leq \sup _{t \in I_{\alpha}^{T}}\left\{\frac{N e_{o}^{\kappa T}\left(\left(L_{1}+\varphi_{0} T L_{3}\right)|x(u)|+L_{2}\left|D_{w}\left[e_{o}^{\kappa u} x(u)\right]\right|+K\right)}{\Gamma_{p}(\gamma)(1-w) t}\right. \\
& \times\left|\int_{0}^{t} \int_{0}^{s} \int_{0}^{z}(s-p z)^{(\gamma-1)} d_{q} u d_{p} z d_{o} s-\int_{0}^{w t} \int_{0}^{s} \int_{0}^{z}(s-p z)^{(\gamma-1)} d_{q} u d_{p} z d_{o} s\right| \\
& +\frac{N e_{o}^{\kappa T}\left|\mathbf{O}_{2}^{*}(f)\right|}{\Gamma_{p}(\gamma)(1-w) t}\left|\int_{0}^{t} \int_{0}^{s}(s-p z)^{(\gamma-1)} d_{p} z d_{o} s-\int_{0}^{w t} \int_{0}^{s}(s-p z)^{(\gamma-1)} d_{p} z d_{o} s\right| \\
& \left.+\left|\mathbf{O}_{3}^{*}(f)\right|\left(\kappa \sum_{n=0}^{\infty} \frac{[n]_{w}}{[n]_{o} !}(\kappa t)^{n-1}\right)\right\} \\
& \leq\left(\lambda\|x\|_{\mathcal{C}}+K\right) \frac{t^{\gamma+1} N[\gamma+2]_{w}}{\Gamma_{p}(\gamma+2)[\gamma+2]_{o}}+\left|\mathbf{O}_{2}^{*}(f)\right| \frac{t^{\gamma} N[\gamma+1]_{w}}{\Gamma_{p}(\gamma+1)[\gamma+1]_{o}} \\
& +\left|\mathbf{O}_{3}^{*}(f)\right|\left(\kappa \sum_{n-1=0}^{\infty} \frac{(\kappa t)^{n-1}}{[n-1]_{o} !}\right) \\
& \leq\left(\lambda\|x\|_{\mathcal{C}}+K\right)\left(\frac{T^{\gamma} N[\gamma+1]_{w}}{\Gamma_{p}(\gamma+2)}\left[\frac{T}{[\gamma+2]_{o}}++\frac{N e_{o}^{\kappa T}}{n[\gamma+1]_{o}}\right]\right. \\
& \left.+\frac{N \kappa\left(e_{o}^{\kappa T}\right)^{2} T^{\gamma}}{\Gamma_{p}(\gamma+2)}\left[\frac{T}{[\gamma+2]_{o}}+\frac{1}{[\gamma+1]_{o}}\right]\right) \\
& =\left(\lambda\|x\|_{\mathcal{C}}+K\right) \Omega_{2} \text {. }
\end{aligned}
$$

Therefore, we obtain

$$
\|F x\|_{\mathcal{C}} \leq\left(\lambda\|x\|_{\mathcal{C}}+K\right)\left(\Omega_{1}+\Omega_{2}\right) \leq(\lambda R+K)\left(\Omega_{1}+\Omega_{2}\right) \leq R .
$$

Hence, we can conclude that $F B_{R} \subset B_{R}$.

Further, considering for any $x, y \in \mathcal{C}$ and $t \in I_{\alpha}^{T}$, letting

$$
|f[x(t)]-f[y(t)]|:=\left|f\left(t, x(t), D_{w}\left[e_{o}^{\kappa t} x(t)\right], \Psi_{\nu} x(t)\right)-f\left(t, y(t), D_{w}\left[e_{o}^{\kappa t} y(t)\right], \Psi_{\nu} y(t)\right)\right|,
$$


we find that

$$
\begin{aligned}
& \mid(F x)(t)-(F y)(t) \mid \\
& \leq \sup _{t \in I_{\alpha}^{T}}\left\{\frac{e_{o}^{-\kappa t}}{\Gamma_{p}(\gamma)} \int_{0}^{t} \int_{0}^{s} \int_{0}^{z} e_{o}^{\kappa s}(s-p z)^{(\gamma-1)} P(z)|f[x(t)]-f[y(t)]| d_{q} u d_{p} z d_{o} s\right. \\
&+ {\left[\frac{M N e_{o}^{-\kappa t}}{m \Gamma_{p}(\gamma) \int_{0}^{T}(T-r s)^{(\theta-1)} d_{r} s} \int_{0}^{T} \int_{0}^{s} \int_{0}^{z} \int_{0}^{y}(T-r s)^{(\theta-1)}(z-p y)^{(\gamma-1)}\right.} \\
&\left.\times|f[x(t)]-f[y(t)]| d_{q} u d_{p} y d_{o} z d_{r} s\right]\left[\frac{N e_{o}^{\kappa(T-t)}}{n \int_{0}^{T}(T-p s)^{(\gamma-1)} d_{p} s} \int_{0}^{T} \int_{0}^{s}(T-p s)^{(\gamma-1)}\right. \\
&\left.\times|f[x(t)]-f[y(t)]| d_{q} u d_{p} s\right]\left[\frac{1}{\Gamma_{p}(\gamma)} \int_{0}^{t} \int_{0}^{s} e_{o}^{\kappa s}(s-p z)^{(\gamma-1)} P(z) d_{p} z d_{o} s-\mathbf{A}\right] \\
&+\frac{N e_{o}^{\kappa T}}{\Gamma_{p}(\gamma)\left[e_{o}^{\kappa(T-t)}-1\right]}\left[\int_{0}^{T} \int_{0}^{s} \int_{0}^{z}(s-p z)^{(\gamma-1)}|f[x(t)]-f[y(t)]| d_{q} u d_{p} z d_{o} s\right. \\
&+\left.\frac{N e_{o}^{\kappa T} \int_{0}^{T} \int_{0}^{s}(T-p z)^{(\gamma-1)} d_{p} z d_{o} s}{n \int_{0}^{T}(T-p s)^{(\gamma-1)} d_{p} s} \int_{0}^{T} \int_{0}^{s}(T-p s)^{(\gamma-1)}|f[x(t)]-f[y(t)]| d_{q} u d_{p} s\right] \\
&\left.\quad \times\left[\int_{0}^{t} e_{o}^{\kappa s} d_{o} s-\mathbf{B}\right]\right\} \\
& \leq \lambda\|x-y\|_{\mathcal{C}} \Omega_{1} .
\end{aligned}
$$

Similarly, we have

$$
\left|D_{w}\left[e_{o}^{\kappa t}(F x)\right](t)-D_{w}\left[e_{o}^{\kappa t}(F y)\right](t)\right| \leq \lambda\|x-y\|_{\mathcal{C}} \Omega_{2}
$$

Consequently, we obtain

$$
\|F x-F y\|_{\mathcal{C}} \leq \lambda\|x-y\|_{\mathcal{C}}\left(\Omega_{1}+\Omega_{2}\right) .
$$

From $\left(\mathrm{H}_{3}\right)$, we can conclude that $F$ is a contraction. Therefore, our proof is completed by using Banach's contraction mapping principle.

The following theorems show the existence of at least one solution to the boundary value problem (1.5) by employing the Krasnoselskii fixed point theorem.

Theorem 3.2 [24] Let $E$ is a bounded closed convex and nonempty subset of a Banach space $X$. If $A, B$ are operators such that:

(i) $A x+B y \in E$ whenever $x, y \in E$,

(ii) $A$ is compact and continuous,

(iii) $B$ is a contraction mapping;

then there exists $z \in E$ such that $z=A z+B z$.

Theorem 3.3 Assume that the condition $\left(\mathrm{H}_{1}\right)-\left(\mathrm{H}_{3}\right)$ of Theorem 3.1 are assumed. In addition we suppose that:

$\left(\mathrm{H}_{4}\right)\left|f\left(t, x, D_{w}\left[e_{o}^{\kappa t} x(t)\right], \Psi_{\nu} x\right)\right| \leq \mu(t)$, for all $\left(t, x, D_{w}\left[e_{o}^{\kappa t} x(t)\right], \Psi_{\nu} x\right) \in I_{\alpha}^{T} \times \mathbb{R} \times \mathbb{R} \times \mathbb{R}$, with $\mu \in C\left(I_{\alpha}^{T}, \mathbb{R}^{+}\right)$. 
Then the boundary value problem (1.5) has at least one solution on $I_{\alpha}^{T}$ if

$$
\Omega_{1}+\Omega_{2}<1
$$

where $\Omega_{1}$ and $\Omega_{2}$ are given by (3.5).

Proof We set $\sup _{t \in I_{\alpha}^{T}}|\mu(t)|=\|\mu\|$, choose a constant

$$
\ell \geq\|\mu\|\left(\Omega_{1}+\Omega_{2}\right)
$$

and let $B_{\ell}=\left\{x \in \mathcal{C}:\|x\|_{\mathcal{C}} \leq \ell\right\}$.

On the given ball $B_{\ell}$, we define the operators $\mathcal{F}_{1}$ and $\mathcal{F}_{2}$ as

$$
\begin{aligned}
\left(\mathcal{F}_{1} x\right)(t)= & \frac{e_{o}^{-\kappa t}}{\Gamma_{p}(\gamma)} \int_{0}^{t} \int_{0}^{s} \int_{0}^{z} e_{o}^{\kappa s}(s-p z)^{(\gamma-1)} P(z) \\
& \times f\left(u, x(u), D_{w}\left[e_{o}^{\kappa u} x(u)\right], \Psi_{\curlywedge} x(u)\right) d_{q} u d_{p} z d_{o} s-e_{o}^{-\kappa t} \mathbf{O}_{1}^{*}(f), \\
\left(\mathcal{F}_{2} x\right)(t)= & e_{o}^{-\kappa t} \mathbf{O}_{2}^{*}(f)\left[\mathbf{A}-\frac{1}{\Gamma_{p}(\gamma)} \int_{0}^{t} \int_{0}^{s} e_{o}^{\kappa s}(s-p z)^{(\gamma-1)} P(z) d_{p} z d_{o} s\right] \\
& +e_{o}^{-\kappa t} \mathbf{O}_{3}^{*}(f)\left[\int_{0}^{t} e_{o}^{\kappa s} d_{o} s-\mathbf{B}\right] .
\end{aligned}
$$

For any $x, y \in B_{\ell}$, since

$$
\begin{aligned}
& \left|\mathcal{F}_{1} x+\mathcal{F}_{2} y\right| \\
& \leq \sup _{t \in I_{\alpha}^{T}}\left\{\frac{\|\mu\| N e_{o}^{-\kappa t}}{\Gamma_{p}(\gamma)} \int_{0}^{t} \int_{0}^{s} \int_{0}^{z} e_{o}^{\kappa s}(s-p z)^{(\gamma-1)} d_{q} u d_{p} z d_{o} s\right. \\
& +\left[\frac{\|\mu\| M N e_{0}^{-\kappa t}}{m \Gamma_{p}(\gamma) \int_{0}^{T}(T-r s)^{(\theta-1)} d_{r} s} \int_{0}^{T} \int_{0}^{s} \int_{0}^{z} \int_{0}^{y}(T-r s)^{(\theta-1)}\right. \\
& \left.\times(z-p y)^{(\gamma-1)} d_{q} u d_{p} y d_{o} z d_{r} s\right] \\
& +\left[\frac{\|\mu\| N e_{o}^{\kappa(T-t)}}{n \int_{0}^{T}(T-p s)^{(\gamma-1)} d_{p} s} \int_{0}^{T} \int_{0}^{s}(T-p s)^{(\gamma-1)} d_{q} u d_{p} s\right] \\
& \times\left[\frac{1}{\Gamma_{p}(\gamma)} \int_{0}^{t} \int_{0}^{s} e_{o}^{\kappa s}(s-p z)^{(\gamma-1)} P(z) d_{p} z d_{o} s+\mathbf{A}\right]+\frac{\|\mu\| N e_{o}^{\kappa T}}{\Gamma_{p}(\gamma)\left[e_{o}^{\kappa(T-t)}-1\right]} \\
& \times\left[\int_{0}^{T} \int_{0}^{s} \int_{0}^{z}(s-p z)^{(\gamma-1)} d_{q} u d_{p} z d_{0} s+\frac{N e_{o}^{\kappa T} \int_{0}^{T} \int_{0}^{s}(T-p z)^{(\gamma-1)} d_{p} z d_{o} s}{n \int_{0}^{T}(T-p s)^{(\gamma-1)} d_{p} s}\right. \\
& \left.\left.\times \int_{0}^{T} \int_{0}^{s}(T-p s)^{(\gamma-1)} d_{q} u d_{p} s\right]\left[\int_{0}^{t} e_{o}^{\kappa s} d_{o} s-\mathbf{B}\right]\right\} \\
& \leq\|\mu\| \Omega_{1} \text {, }
\end{aligned}
$$

and by the same argument as above, we have

$$
\left|D_{w}\left[e_{o}^{\kappa t}\left(F_{1} x\right)\right](t)-D_{w}\left[e_{o}^{\kappa t}\left(F_{2} y\right)\right](t)\right| \leq\|\mu\| \Omega_{2} .
$$


Consequently, we obtain

$$
\left\|F_{1} x-F_{2} y\right\|_{\mathcal{C}} \leq\|\mu\|\left(\Omega_{1}+\Omega_{2}\right)
$$

We can conclude that $\mathcal{F}_{1} x+\mathcal{F}_{2} y \in B_{\ell}$. By the condition (3.3), $\mathcal{F}_{2}$ is a contraction mapping. From the continuity of $f$ and $P$, with the condition $\left(\mathrm{H}_{4}\right)$, the operator $\mathcal{F}_{1}$ is continuous and uniformly bounded on $B_{\ell}$. We next show that $\mathcal{F}_{1}$ is compact. For $t_{1}, t_{2} \in I_{\alpha}^{T}$ where $t_{1} \leq t_{2}$ and $x \in B_{\ell}$, we have

$$
\begin{aligned}
\left|\mathcal{F}_{1} x\left(t_{2}\right)-\mathcal{F}_{1} x\left(t_{1}\right)\right| & \\
\leq & \mid \frac{e_{o}^{-\kappa t_{2}} N\|\mu\|}{\Gamma_{p}(\gamma)} \int_{0}^{t_{2}} \int_{0}^{s} \int_{0}^{z} e_{o}^{\kappa s}(s-p z)^{(\gamma-1)} d_{q} u d_{p} z d_{o} s \\
& \quad-\frac{e_{o}^{-\kappa t_{1}} N\|\mu\|}{\Gamma_{p}(\gamma)} \int_{0}^{t_{1}} \int_{0}^{s} \int_{0}^{z} e_{o}^{\kappa s}(s-p z)^{(\gamma-1)} d_{q} u d_{p} z d_{o} s \mid \\
\leq & \frac{N\|\mu\|}{\Gamma_{p}(\gamma)} \int_{t_{1}}^{t_{2}} \int_{0}^{s} \int_{0}^{z}(s-p z)^{(\gamma-1)} d_{q} u d_{p} z d_{o} s \\
\leq & \frac{N\|\mu\|}{\Gamma_{p}(\gamma+1)} \int_{t_{1}}^{t_{2}} s^{\gamma} d_{o} s=\frac{N\|\mu\|\left|t_{2}^{\gamma+1}-t_{1}^{\gamma+1}\right|}{\Gamma_{p}(\gamma+1)[\gamma+1]_{o}} .
\end{aligned}
$$

We see that the right-hand side of the latter inequality tends to zero if $t_{2} \rightarrow t_{1}$. Therefore, $\mathcal{F}_{1}$ is relatively compact on $B_{\ell}$. Hence, by the Arzelá-Ascoli theorem, we can conclude that $\mathcal{F}_{1}$ is compact on $B_{\ell}$.

We find that all assumptions of Theorem 3.2 are satisfied. Therefore, we can reach the conclusion that the boundary value problem (1.5) has at least one solution on $I_{\alpha}^{T}$. Our proof is completed.

\section{Example}

To illustrate our main result, we provide an example of the boundary value problem of second-order $q$-difference equations with $q$-integral boundary conditions:

$$
\left\{\begin{array}{l}
D_{\frac{1}{2}}\left[\left(2 \pi+e^{10} \sin ^{2}\left(\frac{2 \pi t}{3}\right)\right) D_{\frac{1}{3}}^{\frac{1}{2}}\left(\frac{1}{3}+D_{\frac{1}{4}}\right)\right] x(t) \\
=\frac{e^{-\sin ^{2}\left(\frac{2 \pi t}{3}\right)}}{100+e^{\cos ^{2}\left(\frac{2 \pi t}{3}\right)}} \cdot \frac{|x(t)|+\left|D_{\frac{1}{4}}\left[e_{\frac{1}{4}}^{\frac{3}{3}} x(t)\right]\right|+\left|\Psi \frac{4}{5} x(t)\right|}{1+|x(t)|}, \\
x(0)=x(3) \\
\left.D_{\frac{1}{4}}\left[e_{\frac{1}{4}}^{\frac{t}{3}} x(t)\right]\right|_{t=0}=D_{\frac{1}{4}}\left[e_{\frac{1}{4}}^{\frac{T}{3}} x(T)\right] \\
I_{\frac{3}{4}}^{\frac{2}{5}} \sigma(3) x(3)=\frac{3}{\Gamma_{\frac{3}{4}}\left(\frac{2}{5}\right)} \int_{0}^{3}(1-s)^{\left(-\frac{3}{5}\right)} e^{\cos \left(\frac{2 \pi s}{3}\right)} x(s) d_{\frac{3}{4}} s=0,
\end{array}\right.
$$

where $t \in I_{\frac{1}{60}}^{3}=\left\{3\left(\frac{1}{60}\right)^{n}: n \in \mathbb{N}\right\} \cup\{0,3\}$ and $\Psi_{\frac{4}{5}} x(t)=\int_{0}^{t} \sqrt{t s} \cdot x(s) d_{\frac{4}{5}} s$.

Applying Theorem 3.1, when $q=\frac{1}{2}, p=\frac{1}{3}, o=\frac{1}{4}, r=\frac{3}{4}, w=\frac{1}{4}, v=\frac{4}{5}, \theta=\frac{2}{5}, T=3$, $f\left(t, x, D_{w}\left[e_{o}^{\kappa t} x(t)\right], \Psi_{\nu} x(t)\right)=\frac{e^{-\sin ^{2}\left(\frac{2 \pi t}{3}\right)}}{100+e^{\cos ^{2}\left(\frac{2 \pi t}{3}\right)}} \cdot \frac{|x(t)|+\left|D_{\frac{1}{4}}\left[e_{\frac{1}{4}}^{\frac{t}{3}} x(t)\right]\right|+\left|\Psi_{\frac{4}{5}} x(t)\right|}{1+|x(t)|}, \kappa=\frac{1}{3}, P(t)=\frac{1}{2 \pi+e^{10} \sin ^{2}\left(\frac{2 \pi t}{3}\right)}$, $\sigma(t)=e^{\cos \left(\frac{2 \pi t}{3}\right)}$, and $\varphi_{0}=\sup \{\varphi(t, s)\}=3$. 
Since

$$
\begin{aligned}
& \left|f\left(t, x, D_{w}\left[e_{o}^{\kappa t} x(t)\right], \Psi_{\nu} x(t)\right)-f\left(t, y, D_{w}\left[e_{o}^{\kappa t} y(t)\right], \Psi_{v} y(t)\right)\right| \\
& \quad \leq \frac{(1+9)}{101}|x-y|+\frac{1}{101}\left|D_{\frac{1}{4}}\left[e_{\frac{1}{4}}^{\frac{t}{3}} x(t)\right]-D_{\frac{1}{4}}\left[e_{\frac{1}{4}}^{\frac{t}{3}} y(t)\right]\right|
\end{aligned}
$$

so $\left(\mathrm{H}_{1}\right)$ satisfies with $\lambda=\max \left\{L_{1}+9 L_{3}, L_{2}\right\}=\frac{10}{101}$.

Also, we have $\frac{1}{e} \leq \sigma(t) \leq e$ and $\frac{1}{2 \pi} \leq P(t) \leq \frac{1}{2 \pi+e^{10}}$, then $\left(\mathrm{H}_{2}\right)$ is satisfied with $M=e$, $m=\frac{1}{e}, N=\frac{1}{2 \pi+e^{10}}$, and $n=\frac{1}{2 \pi}$. Moreover, we can show that

$$
\Omega_{1}=0.5544 \text { and } \Omega_{2}=0.0111
$$

\section{We obtain}

$$
\lambda\left(\Omega_{1}+\Omega_{2}\right) \approx 0.0599<1,
$$

which implies that $\left(\mathrm{H}_{3}\right)$ holds. By Theorem 3.1, we can conclude that the above problem (4.1) has a unique solution on $I_{\frac{1}{60}}^{3}$.

\section{Competing interests}

The authors declare that there are no competing interests.

\section{Authors' contributions}

The authors declare that they carried out all the work in this manuscript and read and approved the final manuscript.

\section{Acknowledgements}

This research was funded by King Mongkut's University of Technology North Bangkok. Contract no. KMUTNB-GOV-58-52. The authors would like to thank the anonymous referees for carefully reading the paper and for their comments, which have improved the manuscript.

\section{Received: 9 March 2016 Accepted: 25 May 2016 Published online: 07 June 2016}

\section{References}

1. Jackson, FH: q-Difference equations. Am. J. Math. 32, 305-314 (1970)

2. Carmichael, RD: The general theory of linear q-difference equations. Am. J. Math. 34, 147-168 (1912)

3. Mason, TE: On properties of the solutions of linear q-difference equations with entire function coefficients. Am. J. Math. 37, 439-444 (1915)

4. Kac, V, Cheung, P: Quantum Calculus. Springer, New York (2000)

5. Al-Salam, WA: Some fractional q-integrals and q-derivatives. Proc. Edinb. Math. Soc. 15(2), 135-140 (1966/1967)

6. Agarwal, RP: Certain fractional q-integrals and q-derivatives. Proc. Camb. Philos. Soc. 66, 365-370 (1969)

7. Annaby, MH, Mansour, ZS: q-Fractional Calculus and Equations. Lecture Notes in Mathematics, vol. 2056. Springer, Berlin (2012)

8. Rajković, P, Marinković, S, Stanković, M: Fractional integrals and derivatives in q-calculus. Appl. Anal. Discrete Math. 1(1), 311-323 (2007)

9. El-Shahed, M, Hassan, HA: Positive solutions of q-difference equation. Proc. Am. Math. Soc. 138, 1733-1738 (2010)

10. Ahmad, B, Ntouyas, SK: Boundary value problems for q-difference inclusions. Abstr. Appl. Anal. 2011, Article ID $292860(2011)$

11. Ahmad, B, Ntouyas, SK, Purnaras, IK: Existence results for nonlinear q-difference equations with nonlocal boundary conditions. Commun. Appl. Nonlinear Anal. 19, 59-72 (2012)

12. Ahmad, B, Nieto, Jj: On nonlocal boundary value problems of nonlinear q-difference equations. Adv. Differ. Equ. 2012, 81 (2012)

13. Ahmad, B, Ntouyas, SK: Existence of solutions for nonlinear fractional q-difference inclusions with nonlocal Robin (separated) conditions. Mediterr. J. Math. 10, 1333-1351 (2013)

14. Ahmad, B, Ntouyas, SK, Alsaedi, A, Al-Hutami, H: Nonlinear q-fractional differential equations with nonlocal and sub-strip type boundary conditions. Electron. J. Qual. Theory Differ. Equ. 2014, 26 (2014)

15. Ahmad, B, Nieto, JJ, Alsaedi, A, Al-Hutami, H: Existence of solutions for nonlinear fractional q-difference integral equations with two fractional orders and nonlocal four-point boundary conditions. J. Franklin Inst. 351, $2890-2909$ (2014)

16. Almeida, $\mathrm{R}$, Martins, $\mathrm{N}$ : Existence results for fractional $q$-difference equations of order $\alpha \in] 2,3[$ with three-point boundary conditions. Commun. Nonlinear Sci. Numer. Simul. 19, 1675-1685 (2014) 
17. Pongarm, N, Asawasamrit, S, Tariboon, J, Ntoyas, SK: Multi-strip fractional q-integral boundary value problems for nonlinear fractional q-difference equations. Adv. Differ. Equ. 2014, 193 (2014)

18. Pongarm, $N$, Asawasamrit, S, Tariboon, J: Sequential derivatives of nonlinear $q$-difference equations with three-point q-integral boundary conditions. J. Appl. Math. 2013, Article ID 7605169 (2013)

19. Agarwal, RP, Wang, G, Hobiny, A, Zhang, L, Ahmad, B: Existence and nonexistence of solutions for nonlinear second order q-integro-difference equations with non-separated boundary conditions. J. Nonlinear Sci. Appl. 8, 976-985 (2015)

20. Sitthiwirattham, T: On nonlocal fractional $q$-integral boundary value problems of fractional $q$-difference and fractional $q$-integrodifference equations involving different numbers of order and q. Bound. Value Probl. 2016, 12 (2016)

21. Sitthiwirattham, T, Tariboon, J, Ntouyas, SK: Three-point boundary value problems of nonlinear second-order q-difference equations involving different numbers of q. J. Appl. Math. 2013, Article ID 763786 (2013)

22. Patanarapeelert, $\mathrm{N}$, Sitthiwirattham, $\mathrm{T}$ : Existence results of sequential derivatives of nonlinear $q$-difference equations with a new class of three-point boundary value problems conditions. J. Comput. Anal. Appl. 18(5), 844-856 (2015)

23. Saengngammongkhol, T, Kaewwisetkul, B, Sitthiwirattham, T: Existence results for nonlinear second-order q-difference equations with q-integral boundary conditions. Differ. Equ. Appl. 7(3), 303-311 (2015)

24. Krasnoselskii, MA: Two remarks on the method of successive approximations. Usp. Mat. Nauk 10, 123-127 (1955)

\section{Submit your manuscript to a SpringerOpen ${ }^{\circ}$ journal and benefit from:}

- Convenient online submission

- Rigorous peer review

- Immediate publication on acceptance

- Open access: articles freely available online

High visibility within the field

- Retaining the copyright to your article 